Proceedings of the ASME 2017 International Design Engineering Technical Conferences \&
Computers and Information in Engineering Conference
IDETC2017
August 6-9, 2017, Cleveland, Ohio, USA

DETC2017/DAC-67684

\title{
DRAFT: FORCE DIVERTING HELMET LINER ACHIEVED THROUGH A LATTICE OF MULTI-MATERIAL COMPLIANT MECHANISMS
}

\author{
Vaibhav Gokhale \\ Prasad Tapkir \\ Andres Tovar \\ Department of Mechanical Engineering \\ Indiana University-Purdue University Indianapolis \\ Indianapolis, Indiana \\ vvgokhal@iupui.edu, pbtapkir@iupui.edu, tovara@iupui.edu
}

\begin{abstract}
This work introduces the design of a lattice array of multimaterial compliant mechanisms (LCM) that diverts the impact radial force into tangential forces through the action of elastic hinges and connecting springs. When used as the helmet liner, the LCM liner design has the potential to reduce the risk of head injury through improved impact energy attenuation. The compliant mechanism array in the liner is optimized using a multi-material topology optimization algorithm. The performance of the LCM liner design is compared with the one obtained by expanded polypropylene (EPP) foam, which is traditionally used in sport helmets. An impact test is carried out using explicit, dynamic, nonlinear finite element analysis. The parameters under consideration include the internal energy, the peak linear force, as well as von Mises stress and effective plastic strain distributions. Although there is a small increase in stress and strain values, the simulations show that the maximum internal of the LCM liner design is four times the one of the foam design while the peak linear force is reduced to about half. While the use of the LCM liner design is intended for sports helmets, this design may find application in other energy absorbing structures such as crashworthy vehicle components, blast mitigating structures, and protective gear.
\end{abstract}

\section{INTRODUCTION}

Traumatic brain injuries (TBIs) have a substantial contribution in the total number of deaths and cases of permanent disabilities. According to the U.S. Centers for Disease Control and Prevention, an estimated of 2.5 million people sustain TBIs annually, from which over 52,000 (or 2\%) die [1]. While the TBI phenomenon is not completely understood, there is agreement on two main mechanisms that cause brain injuries. The first mechanism occurs when a moving head is suddenly stopped and the brain continues to move due to its moment of inertia. In this case, the brain breaks the layer of gelatinous fluid surrounding it (cerebrospinal fluid) and bounces against the skull. This causes swelling and injuries of the brain at the outer areas known as coup-countercoup injuries. The type of force responsible for this TBI mechanism is referred to as linear force [2]. An example of a linear force is the head-on impact between two players in American football. The second mechanism occurs when the axons of the neurons at the intersection of regions with different densities are subjected to shearing. These axons break causing neuron death and, eventually, the failure of the neural network. These types of injuries are called diffuse axonal injuries (DAI) and is commonly caused by a rotational force [2]. An example of occurrence of rotational forces is cross punches in boxing from left or right side of the player which cause the head to rotate in the direction of the punch.

\footnotetext{
${ }^{*}$ Address all correspondence to this author.
}

This is the author's manuscript of the article published in final edited form as: 
Based on their applications, today's helmets can be classified into three types: the industrial helmet, the sport helmets, and the combat helmet. The industrial helmets (or hard hats) are classified as Type I for top protection and Type II for lateral protection. Type II hard hats are lined on the inside with a high-density foam. The sport helmets are classified based on the energy attenuation characteristics into one-time use and multiuse impact helmets. Most bike helmets are designed to manage high energy impact and utilize a one-time use crushable foam. On the other hand, sport helmets such as American football helmets are designed to manage repetitive impact and utilize non-crushable foams. These helmets also have more number of other accessories than the bike helmets. Finally, the combat (or ballistic) helmets are the most sophisticated in term of the use of materials, which include different types of polymer composites. Their objectives are very different than those of the other classes of helmets: they are supposed to be bulletproof and provide protection from severe blasts. The focus of this work is on sport multi-use impact helmet, but the methods may be extended to other types of helmets.

Desirable goals in sport helmet design are to prevent intrusion, absorb maximum energy per unit volume (specific energy), and produce a low deceleration pulse [5]. To achieve these goals, sport helmet make companies are constantly incorporating new materials and new designs. Outstanding examples in football and motorcycle helmets include the use of viscoelastic polymers such as butyl nitrate and Zorbium, inflatable liners (Riddell SpeedFlex), air shock absorbers (Schutt Air XP Line), and Omni-directional suspension systems (6D Helmets). Several research groups are currently addressing fundamental design aspects in football helmets [3-5], motorcycle helmets [6-8], and bicycle helmets [9, 10]. Interestingly, specialized helmets are being developed for sports such as pole vault, soccer, lacrosse, and field hockey [11], as well as new sports activities such as ebikes, segways, and electric unicycles [12]. While there is no perfect TBI prevention product on the market, innovative helmet designs have contributed to reducing TBI for many sport practitioners ranging from children to elite athletes. Helmet designs should constantly evolve to cope up with the always-evolving sport requirements.

The availability of super-computing capabilities, advanced design algorithms such as topology optimization, and additive manufacturing (3D printing) technologies offer a unique opportunity to shorten the design cycle time and achieve helmet designs with levels of protection not yet attainable. In this research, we propose the design of a protective structural system for helmets composed of three layers: the outer hard shell, the lattice array of multi-material compliant mechanisms (LCM), and the inner hard shell. The outer hard shell meets the impacting object. The inner hard shell is attached to the comfort foam of the helmet's liner. The sandwiched layer between the outer and inner hard shells is the LCM. The LCM is responsible for absorption of most of the impact energy. It contains an array of cellular compliant mechanisms that diverts the impact forces in multiple radial directions. The LCM liner design is obtained through multi-material topology optimization [13]. The proposed LCM liner design can be manufactured using material jetting (PolyJet) additive manufacturing techniques in which multiple nozzles work simultaneously creating multiple digital materials [14].

\section{DESIGN APPROACH Two-phase design}

The volume where the LCM will be designed is divided into representative unit cells. The function of each unit cell is to divert the radial impact force into multiple tangential output forces (Figure 1).

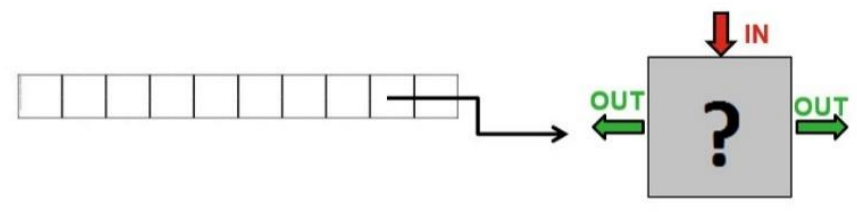

Figure 1: Design domain of the compliant buffer zone discretized into an array unit cells. Each unit cell is subjected to a radial input force

(IN) that is diverted into tangential output forces (OUT).

To accomplish this function, a compliant mechanism is synthesized within each unit cell [15]. Unlike rigid-body mechanisms, compliant mechanisms have flexible members that act as elastic hinges and divert the direction of the load from an input port to a desired output port. In a compliant mechanism, the energy is conserved between input and output ports if friction losses are neglected. One of the most effective strategies to design a compliant mechanism is topology optimization [16]. To this end, the unit cell (design domain) is discretized into $n$ finite elements so that the topology optimization problem is defined as

$$
\begin{array}{cl}
\text { find } & \mathbf{x} \in \mathbb{R}^{n} \\
\max & g_{0}(\tilde{\mathbf{x}})=\mathbf{u}_{\text {in }}(\tilde{\mathbf{x}})^{\mathrm{T}} \mathbf{K}(\tilde{\mathbf{x}}) \mathbf{u}_{\text {out }}(\tilde{\mathbf{x}}) \\
\text { s.t. } & g_{1}(\tilde{\mathbf{x}})=\mathbf{v}^{\mathrm{T}} \tilde{\mathbf{x}}-V=0 \\
& 0 \leq x_{j} \leq 1, \quad j=1, \ldots, n
\end{array}
$$

where $\mathbf{x}$ is the vector of the finite element relative densities, $\tilde{\mathbf{x}}$ is the vector of filtered relative densities, $\mathbf{v}$ is the vector of the finite element volumes, $g_{0}(\tilde{\mathbf{x}})$ is the mutual potential energy function (explained below), and $g_{1}(\tilde{\mathbf{x}})$ is the volume constraint of the compliant mechanism, the volume $V$ controls the maximum amount of the material allowed in the final design. The filtered relative density is defined according the following filter function:

$$
\tilde{x_{J}}=\frac{\sum_{i \in N_{j}} H_{i j} x_{i}}{\sum_{i \in N_{j}} H_{i j}},
$$

where, $N_{j}$ is the neighborhood of $x_{j}$ and $H_{i j}$ is a weighting parameter. The neighborhood of $x_{j}$ is:

$$
N_{j}=\{i: \operatorname{dist}(i, j) \leq R\},
$$

and $R$ is the size or radius of the neighborhood and $\operatorname{dist}(i, j)$ represents the distance between the two discrete locations $i$ and 
$j$. The weighting parameter $H_{i j}$ is defined as a function of $R$ as follows:

$$
H_{i j}=1-\frac{\operatorname{dist}(i, j)}{R}
$$

The stiffness matrix $\mathbf{K}(\tilde{\mathbf{x}})$ in (1) is function of the Young's modulus $E\left(\tilde{x}_{j}\right)$ is interpolated using a modified solid isotropic material with penalization (SIMP) function defined as follows:

$$
E\left(\tilde{x}_{j}\right)=E_{\text {min }}+\tilde{x}_{j}^{q}\left(E_{0}-E_{\text {min }}\right),
$$

where, $E_{0}$ is the Young's modulus of the solid material and $E_{\min }$ is a minimum value; by default, $E_{\min }=10^{-6} E_{0}$. The power $q$ is the so-called penalization power. The result of using $q>1$ is a two-phase material design: $\operatorname{solid}\left(x_{j}=1\right)$ and void $\left(x_{j}=0\right)$.

The mutual potential energy function $g_{0}(\tilde{\mathbf{x}})$ is defined by two independent load cases. In the first load case, a load is applied in the input port while the output port is constrained producing the displacement nodal vector $\mathbf{u}_{i n}(\mathbf{x})$. In the second load case, the load is applied on the output port while the input port is constrained producing the displacement nodal vector $\mathbf{u}_{\text {out }}(\mathbf{x})$. Figure 2 shows the load on the input ports in the radial direction and the load on the output port in the tangential direction. Using symmetry boundary conditions, a quarter of the design domain is considered in the topology optimization problem as shown in Figure 2 (left). The design domain is discretized into $1000 \times 1000$ identical finite elements with unit volume. The allowed volume $V$ corresponds to $25 \%$ of the design domain volume. The penalization power is gradually raised from $q=1$ to $q=3$ to obtain a two-phase solution. The size of the neighborhood is gradually decreased from $R=1.2$ to $R=1.0$ (the size of a finite element) to obtain a binary design. The result of topology optimization is as shown in Figure 2 (right).

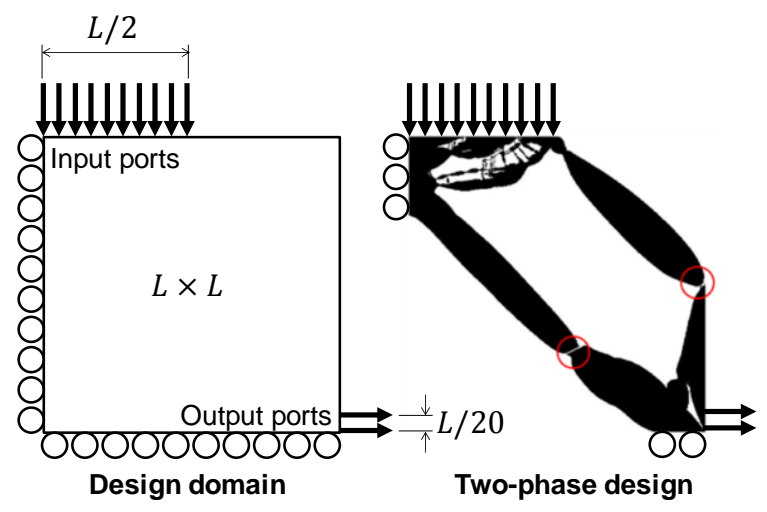

Figure 2: Initial design domain (left) and final two-phase topology (right). The result shows a compliant mechanism with two hinges circled in red.

This design was 3D-printed in acrylonitrile butadiene styrene (ABS) using fused deposition modeling (uPrint, Stratasys, Minnesota) (Figure 3). The elastic hinges are formed by thin members that provide the required flexibility. Unfortunately, for most materials, these thin hinges are unable to withstand deformation without fracture. Due to the lack of mechanical strength and the combined stress concentration at the hinge locations, some of them fail under pressure after a few cycles.
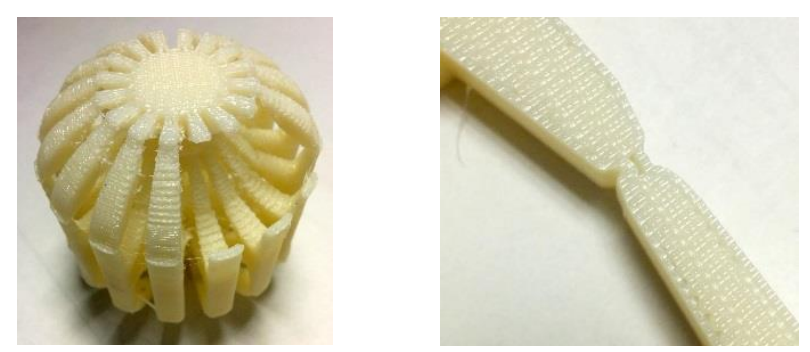

Figure 3: 3D-printed topology in ABS. The picture on the left shows the design of a 3D cellular compliant mechanism with multiple output port radial directions. The picture on the right shows the compliant mechanism hinge in detail.

\section{Multi-material design}

To mitigate the problems associated with thin hinges, this work makes use of the alternating active-phase algorithm for multi-material topology optimization [13]. In this algorithm, the topology optimization (1) is solved alternating two phases at a time. The algorithm is implemented in Matlab [17]. Three material phases are considering: rigid, flexible, and void. The rigid phase is acrylonitrile butadiene styrene (ABS) and the flexible phase is nitrile butadiene rubber (NBR). The volume fractions of the three phases are: $12.5 \%$ for ABS, $12.5 \%$ for NBR, and $75 \%$ for void. The final topology optimization is shown in Figure 4 (left). Using CAD tools, the 2D compliant mechanism is converted in a $3 \mathrm{D}$ axis symmetric compliant mechanism with six output ports as shown in Figure 4 (right). The outer diameter of each compliant mechanism is $25 \mathrm{~mm}$ and the high is also $25 \mathrm{~mm}$. The material on the input and output ports is ABS (red color). The material of the connecting links is in NBR (blue color).

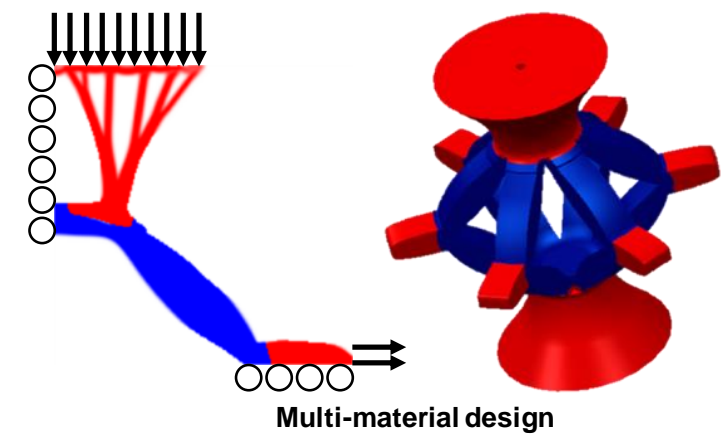

Figure 4: Result of multi-material topology optimization of compliant mechanism with rigid and flexible materials (left), and the 3D axissymmetric compliant mechanism (right).

Adjacent compliant mechanisms are connected through rubber springs made of NBR (Figure 5). The springs have a mean diameter of $5 \mathrm{~mm}$, pitch of $1.9 \mathrm{~mm}$, thickness of $0.5 \mathrm{~mm}$, and length of $10 \mathrm{~mm}$. The design of the rubber spring includes a few passages to facilitate its prototyping using material jetting or stereolithographic additive manufacturing processes. 
Table 1: Material properties of the finite element model.

\begin{tabular}{cccccc}
\hline Material & $\begin{array}{c}\text { Young's modulus } \\
(\mathbf{M P a})\end{array}$ & $\begin{array}{c}\text { Yield strength } \\
\mathbf{( M P a )}\end{array}$ & $\begin{array}{c}\text { Plastic hardening } \\
(\mathbf{M P a})\end{array}$ & $\begin{array}{c}\text { Density } \\
\left(\mathbf{k g} / \mathbf{m}^{\mathbf{3}}\right)\end{array}$ & LS-DYNA model \\
\hline PC & 2,070 & 79.6 & 20.3 & 1200 & 024-PIECEWISE_LINEAR_PLASTICITY \\
ABS & 2,300 & 42.3 & - & 1040 & 024-PIECEWISE_LINEAR_PLASTICITY \\
EPP & 0.69 & 2.7 & - & 86 & 057-LOW DENSITY FOAM \\
NBR & - & - & - & 1150 & 027-MOONEY-RIVLIN_RUBBER \\
\hline
\end{tabular}

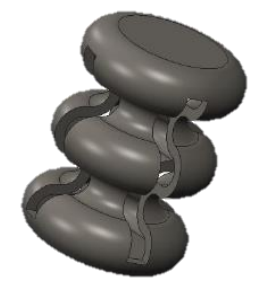

Figure 5: Rubber spring to be used as a connection among compliant mechanisms.

The resulting LCM liner model is sandwiched by two rigid plates made of polycarbonate (PC) of thickness $1 \mathrm{~mm}$ (Figure 6). The finite element model is described in the next section.

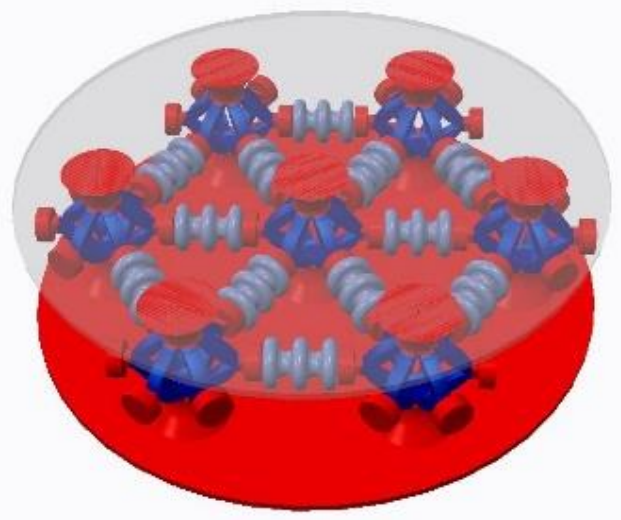

Figure 6: Complete LCM liner design for computational testing.

\section{NUMERICAL MODEL}

Two models are analyzed in this work: the LCM liner design and a traditional foam-based design. The LCM liner design consists of an array of seven, interconnected compliant mechanisms (Figure 6). The foam-based design consists of Expanded Poly Propylene (EPP) foam of density $86 \mathrm{~kg} / \mathrm{m}^{3}$. Both designs are sandwiched by two $1-\mathrm{mm}$ PC plates. The overall dimensions of these models are the same. The material properties of the model are summarized in Table 1. The response of an impacting sphere made of PC (1 mm thickness) is analyzed in each model. The tests are performed using dynamic, explicit finite element analysis with the software LS-DYNA (LSTC, California). The types of finite elements in the model are summarized in Table 2.
Table 2: Finite element types in the LCM linear and benchmark models.

\begin{tabular}{ccc}
\hline Part & Type of element & Material \\
\hline $\begin{array}{c}\text { LCM Compliant } \\
\text { mechanism }\end{array}$ & $\begin{array}{c}\text { Solid 4-noded } \\
\text { tetrahedron } \\
\text { LCM rubber } \\
\text { springs } \\
\begin{array}{c}\text { LCM top and } \\
\text { bottom plates } \\
\text { shell }\end{array}\end{array}$ & ABS and NBR \\
$\begin{array}{c}\text { Foam in traditional } \\
\text { benchmark design }\end{array}$ & $\begin{array}{c}\text { Hexahedron } \\
\text { Belytschko-Tsay } \\
\text { shell }\end{array}$ & NBR \\
Impacting sphere & PC & EPP \\
\hline
\end{tabular}

\section{PERFORMANCE OF THE LCM LINER DESIGN}

The boundary conditions and impact loads of the LCM liner design are shown in Figure 7. The displacement of the LCM's bottom surface is fully constrained, while the top surface receives the impacting sphere traveling at a speed of $5 \mathrm{~m} / \mathrm{s}$. Linear and oblique impacts are considered in this performance study. In the case of linear impact, the direction of impact is parallel to the direction of the axis of compliant mechanism. In the cases of an oblique impact, the direction of impact makes a non-zero angle with the axis of compliant mechanism.

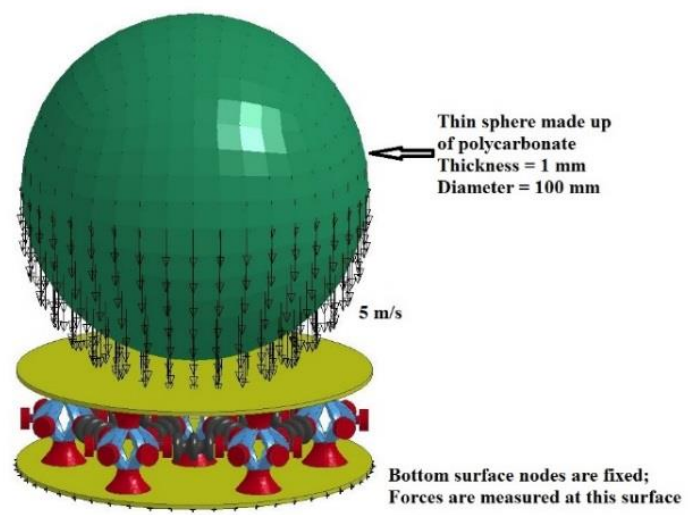

Figure 7: Computational test setup.

For linear impact, three cases are further considered (Figure 8). In the first case (LCM case 1), the impact occurs exactly along the axis of one of the compliant mechanisms. In the second 
case (LCM case 2), the impact is offset from the axis and its location is at the midpoint of the line joining the two adjacent compliant mechanisms in the array. In the third case (LCM case 3 ), the location of the impact is at the centroid of the triangle formed by three adjacent compliant mechanisms.

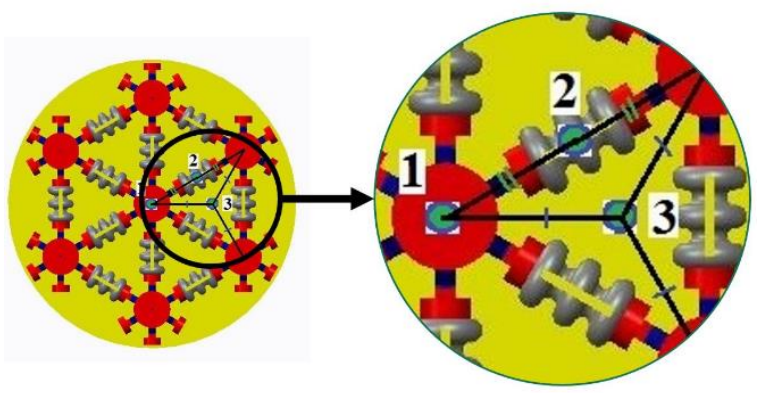

Figure 8: Three load cases of linear impact: (1) on the compliant mechanism, (2) on the rubber spring, and (3) on the centroid.

For oblique impact, two impact angles are considered: $30^{\circ}$ and $60^{\circ}$ (Figure 9). In all simulations, five responses are observed: internal energy, kinetic energy of the impacting sphere, linear reaction force at the bottom surface, von Mises stress on the outer shell, and maximum strain on the outer shell. The results of the LCM liner design are compared to ones of a EPP foam design. The mass of these two models is similar. The mass of the LCM liner design model is $21.29 \mathrm{~g}$ while the mass of the EPP foam design model is $22.22 \mathrm{~g}$. For the EPP foam design, the response does not change for different locations of impact. All results for linear and oblique impact are summarized in the next two sections.
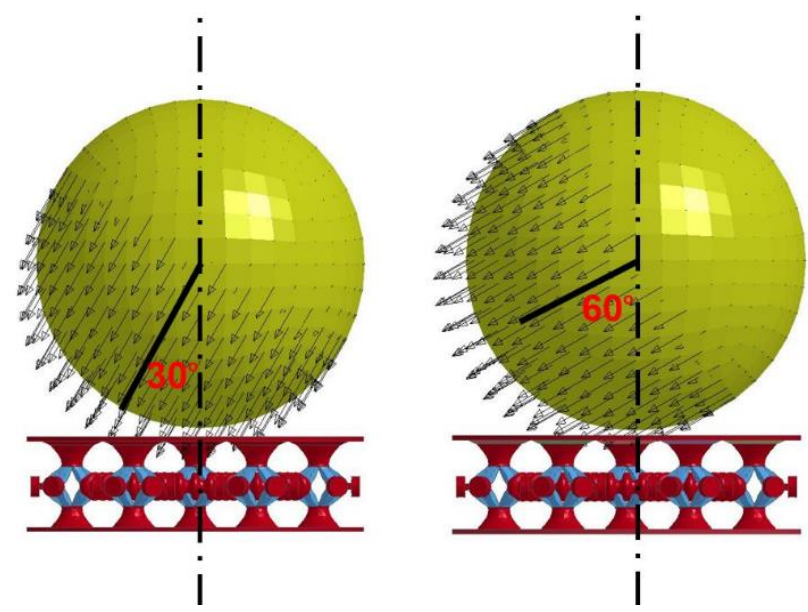

Figure 9: Two cases of oblique impact: $30^{\circ}$ and $60^{\circ}$ impact with respect to the radial axis.

\section{RESULTS OF LINEAR IMPACT}

During the sphere's impact, the protection level of the helmet is quantified through its internal energy as well as the sphere's kinetic energy and the linear reaction force responses over the time. The helmet's internal energy is given by

$$
U(t)=\int_{V} \int_{\boldsymbol{\epsilon}} \boldsymbol{\sigma}(t) \mathrm{d} \boldsymbol{\epsilon} \mathrm{d},
$$

where $\boldsymbol{\sigma}$ and $\boldsymbol{\epsilon}$ are the stress and strain tensors, respectively, and $V$ is the volume. The internal energy response for all helmet material systems (LCM and EPP foam) is shown in Figure 10. Notably, at the end of the simulation, $U(5 \mathrm{~ms})>0$ for the helmet designs due to the deformation of the internal springs. As observed in all load cases, the maximum internal energy of the LCM liner design is $300 \%$ higher than the one of the foam design (Table 3).

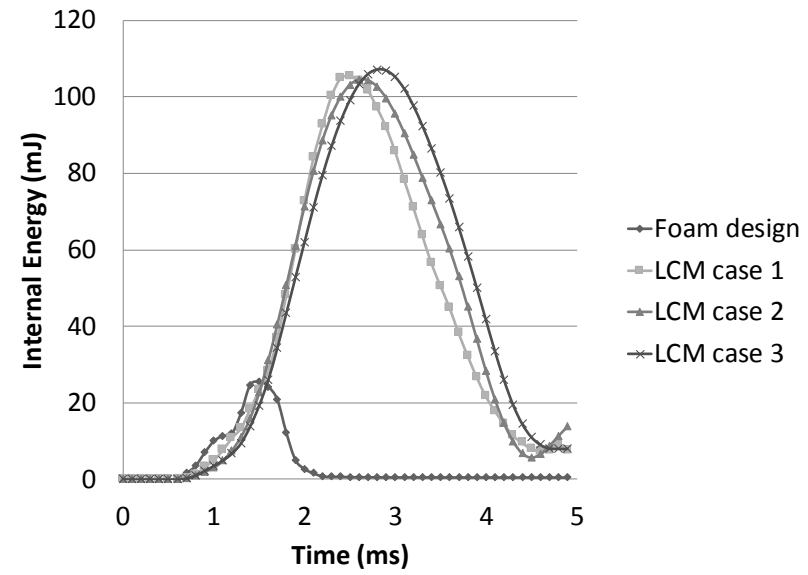

Figure 10: Internal energy as a function of time for linear impact.

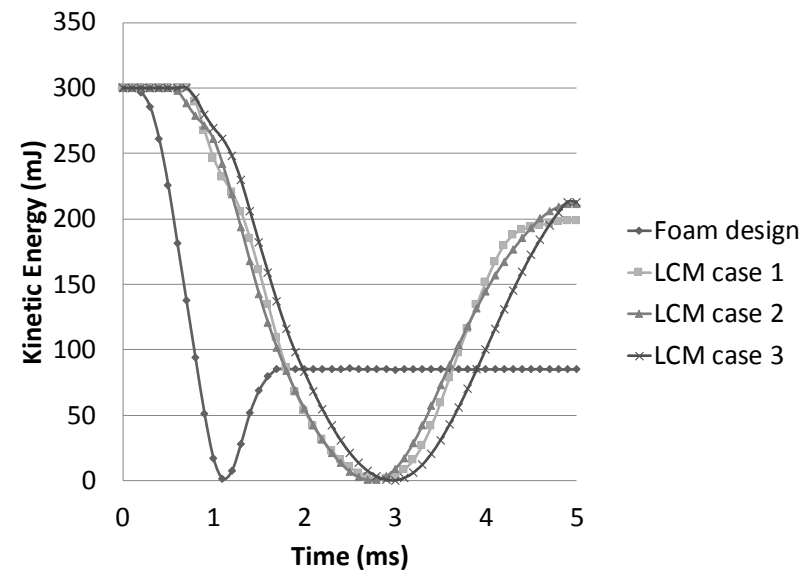

Figure 11: Kinetic energy of the impacting sphere as a function of time for linear impact.

The impacting sphere's kinetic energy responses can be approximated using the translational component as follows:

$$
E_{k}(t)=\frac{1}{2} m v(t)^{2} \text {. }
$$

The kinetic energy responses for the three LCM cases are presented in Figure 11. The initial kinetic energy $E_{k}(0)=0.3 \mathrm{~J}$ (before impact) is the same in all cases. After the impact, the lowest value $E_{k}\left(t^{*}\right)=0$ is achieved at different $\Delta t$ when the sphere comes to rest and the helmet reaches its maximum deformation. The kinetic energy's final value is reached at 
$E_{k}(5 \mathrm{~ms})$ (after impact). The difference between the initial and final values, $\Delta E_{k}=E_{k}(0)-E_{k}(5 \mathrm{~ms})$, quantifies the total of energy transferred from the sphere to the helmet. This value is lower for the LCM liner design. In addition, the time to reach the maximum deformation $t^{*}$ is about $70 \%$ higher for the LCM liner design, which translates into lower linear reaction force.

The linear reaction force $F_{r}$ (Figure 12) causes a linear momentum in the radial direction. The linear momentum is defined as

$$
\begin{aligned}
& \int_{0}^{t} \mathbf{F}(t) d t=m\left(\mathbf{v}(t)-\mathbf{v}_{0}\right) \\
& \quad \therefore \int_{0}^{t} F_{r}(t) d t=m\left(v(t)-v_{0}\right) .
\end{aligned}
$$

In all load cases, the peak linear reaction force occurs at the point of maximum deformation. The peak linear reaction force of the LCM liner design is $50 \%$ lower than the one of the foam design (Table 3).

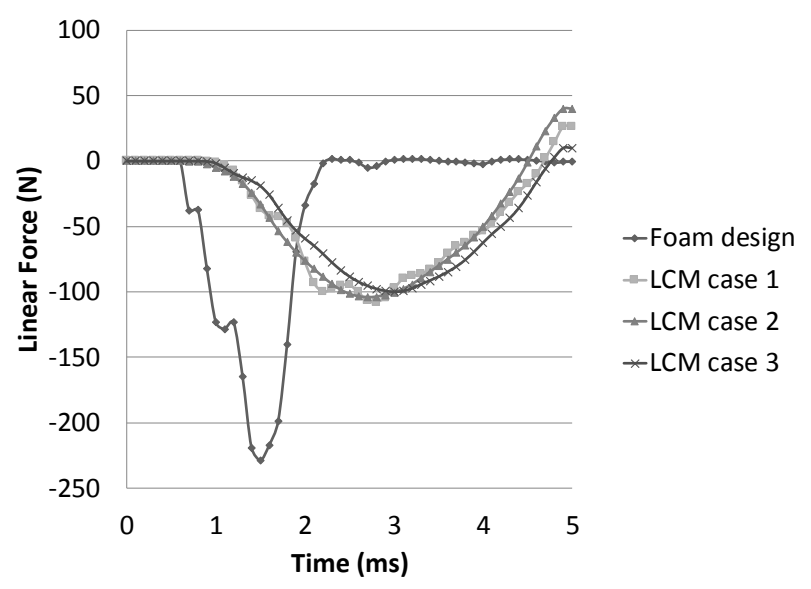

Figure 12: Linear reaction force as a function of time for linear impact.

The maximum von Mises stress $\sigma_{\max }^{\prime}$ on the outer shell tends to be higher in the LCM liner design. In the worst case (LCM case 3 ), the maximum von Mises stress is $23 \%$ higher than the one of the foam design (Figure 13). However, in the best condition (LCM case 1), the maximum von Mises stress is about $6.7 \%$ lower than the one of the foam design. In either case, the von Mises stress is very low to cause damage the outer shell (Table 3).

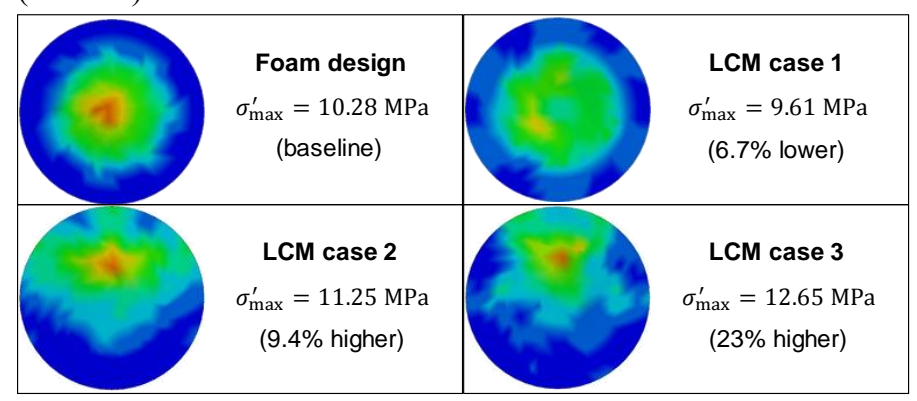

Figure 13: Fringes and maximum von Mises stress values $\sigma_{\max }^{\prime}$ on the outer shell for linear impact.
According to the distortion energy theory, the safety factor against yield is given by

$$
n=\frac{S_{y}}{\sigma^{\prime}}
$$

where $\sigma^{\prime}$ is the von Mises stress and $S_{y}$ is the yield strength. For PC, $S_{y}=79.6 \mathrm{MPa}$ (Table 1). In all LCM load cases, the factor of safety $n$ is greater than 6.29. For a glassy material, such as PC, additional failure criteria include maximum deformation and elongation at break [18]. The elongation at break, adopted in this work, corresponds to the ratio of the change in length after breakage to the initial length. This is also referred to as a fracture strain. For PC, the fracture strain is about $100 \%$ [19]. For the LCM liner design, the maximum strain of the outer shell varies between $42.22 \%$ (LCM case 1 ) to $60.21 \%$ (LCM case 3 ); this represents a deformation at break safety factor from 2.47 to 1.66 . For the foam design, the maximum strain is $47.48 \%$, which carries a safety factor of 2.10. Table 3 summarizes the values of maximum strain of the outer shell.

Table 3: Analysis results considering the traditional EPP foam helmet design and the LCM liner design with three different load cases (Figure 8).

\begin{tabular}{ccccc}
\hline Response & EPP foam & Case 1 & $\begin{array}{c}\text { LCM } \\
\text { Case 2 }\end{array}$ & Case 3 \\
\hline $\begin{array}{c}\text { Max internal } \\
\text { energy (mJ) }\end{array}$ & 25.5 & 105.4 & 104.5 & 107.1 \\
$\begin{array}{c}\text { Time to max. } \\
\text { deformation } \\
(\mathrm{ms})\end{array}$ & 1.1 & 2.8 & 2.7 & 3.0 \\
$\begin{array}{c}\text { Peak linear } \\
\text { force (N) }\end{array}$ & 228.9 & 107.8 & 104.1 & 100.0 \\
$\begin{array}{c}\text { Max. von } \\
\text { Mises stress } \\
(\text { MPa })\end{array}$ & 10.28 & 6.61 & 11.25 & 12.65 \\
$\begin{array}{c}\text { Max. Strain } \\
(\%)\end{array}$ & 47.48 & 42.22 & 54.34 & 60.21 \\
\hline
\end{tabular}

\section{RESULTS OF OBLIQUE IMPACT}

The internal energy of the material system as a function of time is given in Figure 14. The results include impact angles at $0^{\circ}$ (linear impact), $30^{\circ}$, and $60^{\circ}$. Due to the compliant nature of the proposed design, the internal energy of the LCM material system is considerably higher than one of the foam design for all impact angles (Table 4): for $30^{\circ}$ is $900 \%$ higher and for $60^{\circ}$ is $478 \%$ higher. 


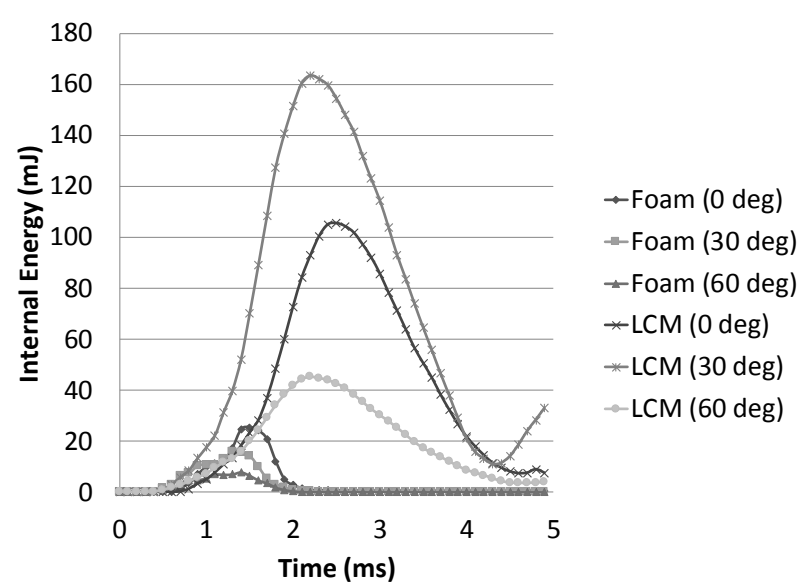

Figure 14: Internal energy as a function of time for oblique impact.

The kinetic energy of the impacting sphere as a function of time is given in Figure 15. For an oblique impact, kinetic energy responses correspond to the LCM case 1. This kinetic energy includes both translational and rotational components:

$$
\begin{aligned}
E_{k}(t) & =\frac{1}{2} m v(t)^{2}+\frac{1}{2} \boldsymbol{\omega}^{\mathrm{T}} \mathbf{I} \boldsymbol{\omega} \\
& =\frac{1}{2} m v(t)^{2}+\frac{1}{2} I \omega(t)^{2},
\end{aligned}
$$

where the angular velocity vector $\boldsymbol{\omega}$ is aligned to the axis perpendicular to the radial-tangential plane so that $I$ is the moment of inertia about the axis of rotation and $\omega(t)$ is the angular velocity of the sphere. In the case of oblique impact, the kinetic energy responses show a similar trend as the ones of linear impact; in other words, the final kinetic energy of the LCM liner design is higher than the ones of the foam design for all impact angles. The time to reach maximum deformation (zero kinetic energy) in the LCM liner design is $90 \%$ higher than the one of the foam design (Table 4).

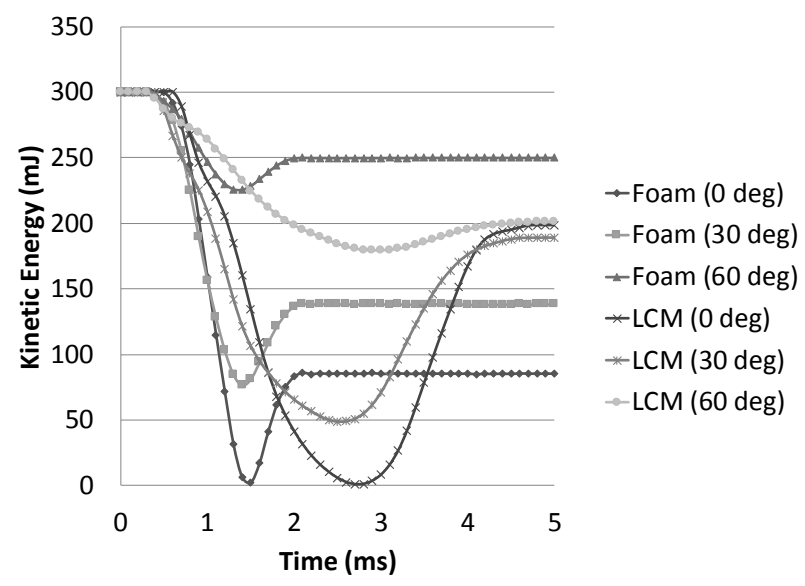

Figure 15: Kinetic energy vs. time for impact at $0^{\circ}, 30^{\circ}$, and $60^{\circ}$.

The increased time to reach maximum deformation produces small linear reaction forces. A contributing factor to this desirable response is the smooth reaction forces exerted by the springs on the sphere via compliant mechanisms. Figure 16 shows the trend of reaction force over the time of impact. The magnitude of the peak linear force of the LCM liner design is $45 \%$ lower than the one of the foam design (Table 4).

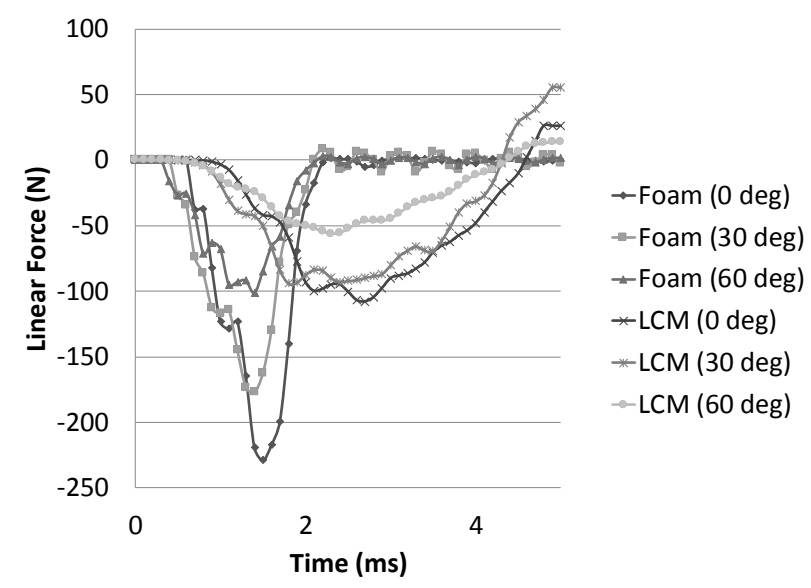

Figure 16: Linear force vs. time for oblique impact at $0^{\circ}, 30^{\circ}$, and $60^{\circ}$.

For the LCM liner design, the maximum von Mises stress on the outer shell is greater than the one of the foam design for all impact angles (Figure 17). Nonetheless, these values are substantially less when compared to the cases of the linear impact. Consequently, the factor of safety is greater than 8.07 in each case (Table 4).

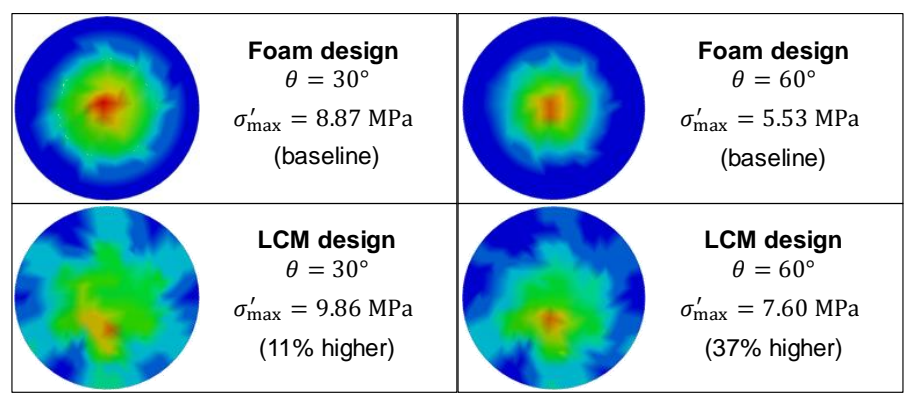

Figure 17: Fringes and maximum von Mises stress values $\sigma_{\max }^{\prime}$ on the outer shell for oblique impact at angles $\theta=30^{\circ}$ and $60^{\circ}$.

The maximum strain of the LCM liner design is $30 \%$ greater than the one of the foam design (Table 4). However, for oblique impact, the numerical value of maximum strain is less than the critical value in the case of linear impact. In summary, while stresses and strains on the outer shell are higher in the LCM liner design, this design offers much better protection than the foam design: higher internal energy and lower peak linear force. 
Table 4: Values of comparison parameters under consideration for oblique impact at $0^{\circ}, 30^{\circ}$, and $60^{\circ}$.

\begin{tabular}{ccccccc}
\hline Response & \multicolumn{3}{c}{ EPP foam } & & \multicolumn{3}{c}{ LCM } \\
& $0^{\circ}$ & $30^{\circ}$ & $60^{\circ}$ & $0^{\circ}$ & $30^{\circ}$ & $60^{\circ}$ \\
\hline $\begin{array}{c}\text { Max internal } \\
\text { energy (mJ) }\end{array}$ & 25.5 & 15.8 & 7.8 & 105.4 & 163.3 & 45.1 \\
$\begin{array}{c}\text { Time to max. } \\
\text { deformation } \\
\text { (ms) }\end{array}$ & 1.5 & 1.4 & 1.4 & 2.7 & 2.5 & 3 \\
$\begin{array}{c}\text { Peak linear } \\
\text { force (N) } \\
\text { Max. von }\end{array}$ & 228.9 & 176.9 & 101.3 & 107.7 & 93.8 & 55.9 \\
$\begin{array}{c}\text { Mises stress } \\
(\text { MPa })\end{array}$ & 10.28 & 8.87 & 5.53 & 6.61 & 9.86 & 7.60 \\
$\begin{array}{c}\text { Max. Strain } \\
(\%)\end{array}$ & 47.48 & 36.89 & 35.61 & 42.22 & 53.37 & 50.92 \\
\hline
\end{tabular}

\section{SUMMARY AND CONCLUSION}

This work presents the design process and computational testing of a material system suitable for sport helmets. This material system is composed of a lattice array of multi-material compliant mechanisms (LCM). The LCM design diverts the impact radial force into tangential forces through the action of elastic hinges and connecting springs. Each multi-material compliant mechanism was synthesized using a 2D multi-material topology optimization algorithm. The algorithm optimally distributed three material phases: rigid, flexible, and void. Using CAD tools, this design is converted into a $3 \mathrm{D}$ axis symmetric design with six output ports. The lattice array is form by connecting compliant mechanisms through rubber springs. The resulting LCM is sandwiched by two polycarbonate (PC) plates to be computationally tested using a dynamic nonlinear finite element analysis (LS-DYNA). A traditional EPP foam helmet design is used as a benchmark. An impacting PC sphere is used in these simulations.

Considering the anisotropic nature of the LCM design, multiple simulations are performed by varying location and direction of the impact. The computational test included linear impact $\left(0^{\circ}\right.$ impact angle) and oblique impact $\left(30^{\circ}\right.$ and $60^{\circ}$ impact angles). The impact simulations demonstrated several advantages of the LCM design over the EPP foam design. First, while the mass of the LCM design (21.29 $\mathrm{g}$ ) is a lower than the one of the EEP foam design (22.22 g), the amount of internal energy absorbed by the LCM is considerably higher than the one absorbed by the EPP foam design: 300\% higher under linear impact and over $478 \%$ higher under oblique impact. Second, the linear force exerted during the impact is at least $45 \%$ lower for the LCM design when compared to the EPP foam design. Since the linear force is directly responsible for producing acceleration on the head, the use of the LCM design in helmets may reduce the risk of head injuries. Third, for certain impact locations, the maximum von Mises stress and maximum stress on the LCM's may be higher than the one of the EPP foam's outer shell's; however, the corresponding safety factors associated with distortion energy (von Mises stress) and deformation at break (strain) are found to be 1.66 or higher.

The LCM design can be tailored by varying the spring constants of the compliant mechanisms and the connecting springs. This can be achieved through resizing and/or material substitution. Considering the manufacturability of the LCM design, the filter radius of the optimization algorithm can be tuned to avoid messy designs and corresponding manufacturing constraints. The prototype of the helmet design can be built using additive manufacturing. Thus, the corresponding cost can be evaluated and compared to the cost of other existing helmet designs. Additional testing on full helmet design are still required. Parameters such as Head Injury Criteria (HIC), Severity Index (SI) as well as linear and angular rotation at the neck must be analyzed.

\section{ACKNOWLEDGMENTS}

The Sports Innovation Institute at IUPUI supported this research effort. Any opinions, findings, conclusions, and recommendations expressed in this investigation are those of the writers and do not necessarily reflect the views of the sponsors.

\section{REFERENCES}

[1] Prevention, C.f.D.C.a., Report to Congress on Traumatic Brain Injury in the United States: Epidemiology and Rehabilitation. 2015, National Center for Injury Prevention and Control; Division of Unintentional Injury Prevention: Atlanta, GA.

[2] Petraglia, A., J. Bailes, and A. Day, Handbook of Neurological Sports Medicine: Concussion and Other Nervous System Injuries in the Athlete. 2014: Human Kinetics.

[3] Moss, W.C., M.J. King, and E.G. Blackman, Towards reducing impact-induced brain injury: Lessons from a computational study of army and football helmet pads. Computer Methods in Biomechanics and Biomedical Engineering, 2014. 17(11): p. 1173-1184.

[4] Johnson, K.L., et al., Constrained topological optimization of a football helmet facemask based on brain response. Materials and Design, 2016. 111: p. 108-118.

[5] Smith, T.A., et al., Angular head motion with and without head contact: implications for brain injury. Sports Engineering, 2015. 18(3): p. 165-175.

[6] Shuaeib, F.M., et al., A new motorcycle helmet liner material: The finite element simulation and design of experiment optimization. Materials and Design, 2007. 28(1): p. 182-195.

[7] Halimi, M.T., M.B. Hassen, and F. Sakli, Design of a novel comfort liner for a motorcycle helmet. International Journal of Sustainable Engineering, 2012. 5(2): p. 128-134.

[8] Blanco, D.H., A. Cernicchi, and U. Galvanetto, Design of an innovative optimized motorcycle helmet. Proceedings of the Institution of Mechanical Engineers, Part P: Journal of 
Sports Engineering and Technology, 2014. 228(2): p. 95110.

[9] Mills, N.J. and A. Gilchrist, Bicycle helmet design. Proceedings of the Institution of Mechanical Engineers, Part L: Journal of Materials: Design and Applications, 2006. 220(4): p. 167-180.

[10] Soe, S.P., et al., Feasibility of optimising bicycle helmet design safety through the use of additive manufactured TPE cellular structures. International Journal of Advanced Manufacturing Technology, 2015. 79(9-12): p. 1975-1982.

[11] Johnson, K. Princeton schools to become the first in state to require headgear for soccer, lacrosse and field hockey players. 2013; Available from: http://www.nj.com/mercer/index.ssf/2013/08/princeton_sc hools become the first in the state to require headgea r_for_soccer_lacrosse_and_field_ho.html.

[12] Helmets.org. Helmets for Many Activities. 2016; Available from: http://www.bhsi.org/other.htm.

[13] Tavakoli, R. and S.M. Mohseni, Alternating active-phase algorithm for multimaterial topology optimization problems: A 115-line MATLAB implementation. Structural and Multidisciplinary Optimization, 2014. 49(4): p. 621642.

[14] Gaynor, A.T., et al., Multiple-Material Topology Optimization of Compliant Mechanisms Created Via
PolyJet Three-Dimensional Printing. Journal of Manufacturing Science and Engineering, Transactions of the ASME, 2014. 136(6).

[15] Gokhale, V.V., et al. Design of an Advanced Layered Composite for Energy Dissipation using a 3D-Lattice of Micro Compliant Mechanism. in SAE 2016 World Congress and Exhibition, April 12, 2016 - April 14, 2016. 2016. Detroit, MI, United states: SAE International.

[16] Bendsoe, M.P. and O. Sigmund, Topology Optimization: Theory, Methods, and Applications. 2003: Springer Berlin Heidelberg.

[17] Liu, K. and A. Tovar, An efficient 3D topology optimization code written in Matlab. Structural and Multidisciplinary Optimization, 2014. 50(6): p. 1175-1196.

[18] Klompen, E.T.J., et al., Quantitative prediction of longterm failure of polycarbonate. Macromolecules, 2005. 38(16): p. 7009-7017.

[19] Inberg, J.P.F. and R.J. Gaymans, Polycarbonate and cocontinuous polycarbonate/ABS blends: Influence of specimen thickness. Polymer, 2002. 43(13): p. 3767-3777. 